Simulation of model overfit in variance explained with genetic data 1

\title{
Simulation of model overfit in variance explained with genetic data
}

\author{
Jaime Derringer, $\mathrm{PhD}^{1}$
}

Submitted draft as of 8 April 2019

Simulation script available at: https://osf.io/t5jbs/

${ }^{1}$ Department of Psychology, University of Illinois at Urbana Champaign, Champaign, IL, USA

Corresponding Author: Jaime Derringer, Department of Psychology, University of Illinois at Urbana-Champaign, 603 East Daniel St., Champaign, IL 61820, USA. Phone: 217-300-6629, Fax: 217-244-5876; Email: jderr@illinois.edu

Conflict of Interest: The author declares that she has no conflict of interest. 
Simulation of model overfit in variance explained with genetic data 2

\section{Abstract}

Two recent papers, and an author response to prior commentary, addressing the genetic architecture of human temperament and character claimed that "The identified SNPs explained nearly all the heritability expected". The authors' method for estimating heritability may be summarized as: Step 1: Pre-select SNPs on the basis of GWAS $p<0.01$ in the target sample. Step 2: Enter target sample genotypes (the pre-selected SNPs from Step 1) and phenotypes into an unsupervised machine learning algorithm (Phenotype-Genotype Many-to-Many Relations Analysis, PGMRA) for further reduction of the set of SNPs. Step 3: Test the sum score of the SNPs identified from Step 2, weighted by the GWAS regression weights estimated in Step 1, within the same target sample. The authors interpreted the linear regression model $\mathrm{R}^{2}$ obtained from Step 3 as a measure of successfully identified heritability. Regardless of the method applied to select SNPs in Step 2, the combination of Steps 1 and 3, as described, causes inflation of the estimated effect size. The extent of this inflation is demonstrated here, where random SNP selection and polygenic scoring from simulated random data recovered effect sizes similar to those reported in the original empirical papers.

\section{Background}

An author response [1] to my previous commentary [2] claimed that the application of PGMRA [3] in parallel papers on the genetics of character [4] and temperament [5] was not subject to concerns about overfitting. Unlike polygenic scoring, the goal was not to identify a generalizable combination of genetic variants to apply to outside samples. Rather, the stated goal was to estimate the heritability in the phenotype that could be attributed to the identified SNPs. The 
Simulation of model overfit in variance explained with genetic data 3

method for estimating what was interpreted as heritability (as illustrated in the author response, Figure $1[1])$ may be summarized as:

Step 1: Pre-select SNPs on the basis of GWAS $p<0.01$ in the target sample.

Step 2: Enter target sample genotypes (the pre-selected SNPs from Step 1) and phenotypes into PGMRA [3] for further reduction of the set of SNPs.

Step 3: Test the sum score of the SNPs identified from Step 2, weighted by the GWAS regression weights estimated in Step 1, within the same target sample.

The authors interpreted the linear regression model $R^{2}$ obtained from Step 3 as a measure of successfully identified heritability. Regardless of the method applied to select SNPs in Step 2 whether it is global or local, data-driven $[4,5]$ or based on theory $[6,7]$ - the combination of Steps 1 and 3, as described, causes inflation of the estimated effect size.

\section{Method}

To demonstrate the extent of effect size inflation resulting from the above-described method, I present a simulation in which random genotypes and phenotypes were generated and Steps 1 and 3 were applied as described by the authors $[1,4,5]$. Here, however, Step 2 was instead executed as random selection of SNPs for inclusion in the score. The entire process, plus cross-validation for comparison, was performed 1000 times per each of 100 simulated data sets, each comprised of 700000 SNPs, with discovery and replication sample sizes of 2000 and 200, respectively. Given that the genotypes, phenotypes, and selected SNPs were entirely random, a correct estimate of the effect should be essentially zero, and any deviation from zero 
Simulation of model overfit in variance explained with genetic data 4

in the estimate of variance explained may be taken as an indicator of model bias, not true heritability.

The extent of inflation in the score is dependent on the number of included predictors. For the current simulation, the number of SNPs included in each random score was set equal to the number of SNPs remaining after pruning for linkage disequilibrium (LD, using a restrictive threshold of $r^{2}>10 \%$ ) the SNPs identified by PGMRA in the largest sample in the character paper (Finns, $N=2149$ [4]). LD structure was estimated in the 1000 Genomes phase 3 Finnish (FIN) reference population [8]. This process yielded an estimate of 359 independent SNPs as an appropriate comparison to the original paper results [4]. The full script for this simulation is available at: https://osf.io/t5jbs/. Simulation and analysis used R [9] and Plink [10].

\section{Result}

The result of these simulations are presented in Figure 1. Within sample scoring, where the identification of SNPs and their effects was estimated and tested within a single sample as described $[1,4,5]$ yielded an average estimate of $60 \%$ variance explained $(S D=0.008)$. This is not less than the variance explained reported by the authors in their non-random datasets $(57 \%$ and $48 \%$ for the Finnish samples applied to character and temperament, respectively [4,5]). When the same randomly selected scores were applied in a cross-validated manner (that is, identified within one sample, tested in an another), the effect size essentially (and correctly, given the random nature of the data) dropped to zero $(\mathrm{M}=0.5 \%, \max =9 \%)$. Although the authors noted that such cross-validated effect sizes may be reduced in the presence of heterogeneity across samples [1], the current simulation generated the sets of genotypes and 
Simulation of model overfit in variance explained with genetic data 5

phenotypes for the discovery and replication samples simultaneously, so that systematic or structural between-sample heterogeneity would not be a likely explanation for the reduction in effect size demonstrated here.

\section{Conclusion}

The observed inflation of the effect size is caused by the combination of Steps 1 and 3, where predictors were selected and tested within the same sample. In no way is this the result of unique properties of any additional selection procedures that may take place in Step 2 (whether PGMRA [3] or otherwise), nor is model overfitting in this manner a problem that is unique to issues of genetic data or machine learning. Although technical capacities and computational models may increase in complexity, such advances do not necessarily overcome the basic requirements of model estimation and evaluation in general. The current simulation does not explore the potential utility of PGMRA for identifying promising sets of associated SNPs.

However, the extent of inflation in the described estimate of "heritability" (the within-sample variance explained; as reported in the abstracts of both original papers [4,5]) is so substantial as to be uninterpretable. 
Simulation of model overfit in variance explained with genetic data 6

\section{References}

1. Zwir I, Mishra P, Del-Val C, Gu CC, de Erausquin GA, Lehtimäki T, Cloninger CR. Uncovering the complex genetics of human personality: response from authors on the PGMRA Model. Mol Psychiatry. 2019;(in press).

https://doi.org/10.1038/s41380-019-0399-z.

2. Derringer J. Explaining heritable variance in human character. bioRxiv. 2018;446518. https://doi.org/10.1101/446518.

3. Arnedo J, del Val C, de Erausquin GA, Romero-Zaliz R, Svrakic D, Cloninger CR, et al. PGMRA: A web server for (Phenotype X Genotype) many-to-many relation analysis in GWAS. Nucleic Acids Res. 2013;41(Web Server issue):W142-9.

4. Zwir I, Arnedo J, Del-Val C, Pulkki-Råback L, Konte B, Yang SS et al. Uncovering the complex genetics of human character. Mol Psychiatry. 2018;(in press). https://doi.org/10.1038/s41380-018-0263-6.

5. Zwir I, Arnedo J, Del-Val C, Pulkki-Raback L, Konte B, Yang SS et al. Uncovering the complex genetics of human temperament. Mol Psychiatry. 2018;(in press). https://doi.org/10.1038/s41380-018-0264-5.

6. Derringer J, Krueger RF, Dick DM, Saccone S, Grucza RA, Agrawal A, et al. Predicting sensation seeking from dopamine genes: A candidate-system approach. Psychol Sci. 2010;21:1282-1290.

7. Powell JE, Zietsch BP. Predicting sensation seeking from dopamine genes: Use and misuse of genetic prediction. Psychol Sci. 2011;22:413-415.

8. Sudmant PH, Rausch T, Gardner EJ, Handsaker RE, Abyzov A, Huddleston J, et al. An integrated map of structural variation in 2,504 human genomes. Nature. 2015;526:75-81. 
bioRxiv preprint doi: https://doi.org/10.1101/598904; this version posted April 10, 2019. The copyright holder for this preprint (which was not certified by peer review) is the author/funder, who has granted bioRxiv a license to display the preprint in perpetuity. It is made available under aCC-BY-NC-ND 4.0 International license.

Simulation of model overfit in variance explained with genetic data 7

9. R Core Team. R: A language and environment for statistical computing. R Foundation for Statistical Computing, Vienna, Austria. 2018. URL https://www.R-project.org/.

10. Purcell S, Change C. PLINK v1.90b4.4 64-bit. 21 May 2017. URL www.cog-genomics.org/plink/1.9/. 
Simulation of model overfit in variance explained with genetic data 8

Figure 1. $R^{2}$ from 100000 simulations of random sets of 359 SNPs scored and applied within the discovery sample ( $N=2000$, white bars) versus cross-validated in an independent replication sample ( $\mathrm{N}=200$, grey bars). Full script available at: https://osf.io/t5jbs/.

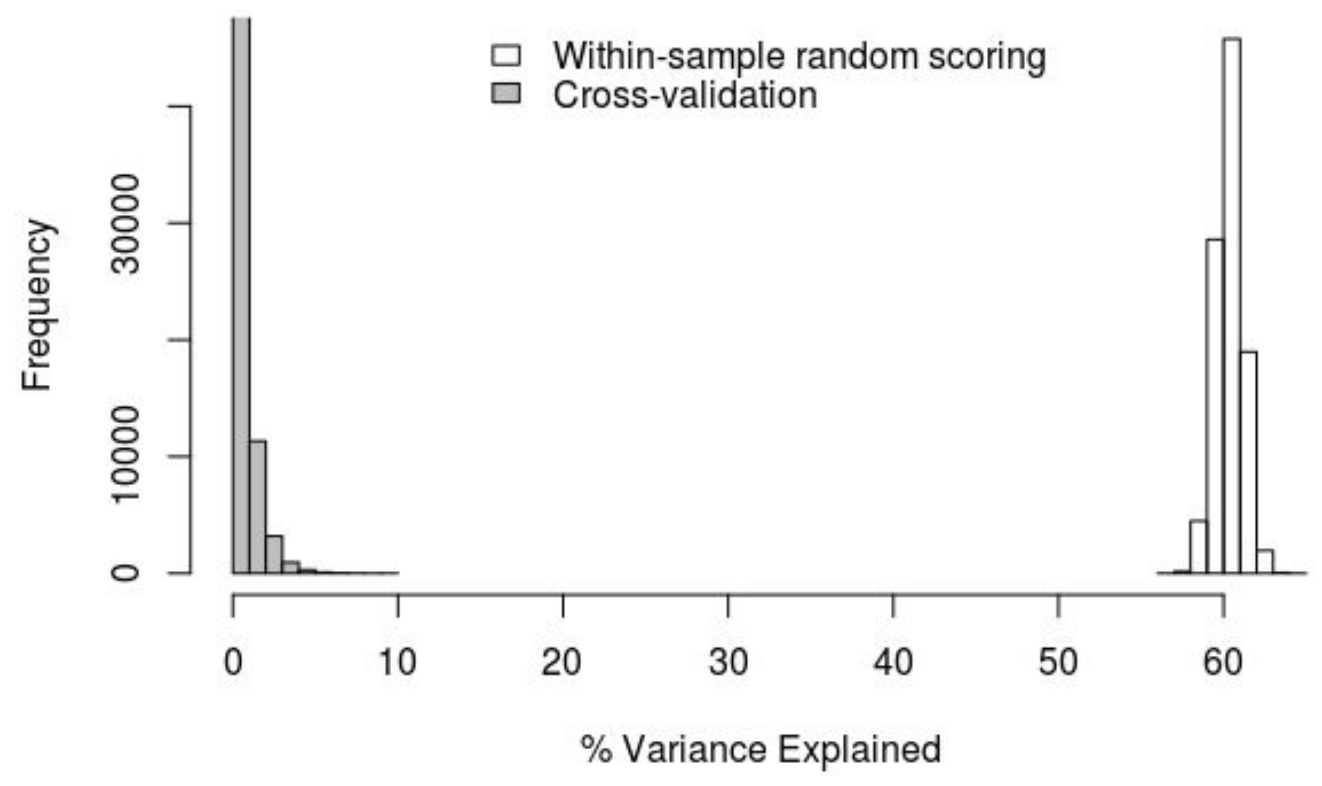

